\title{
A community feasibility study of a cooking behavior intervention in African-American adults at risk for cardiovascular disease: DC COOKS (DC Community Organizing for Optimal culinary Knowledge Study) with Heart
}

Nicole Farmer ${ }^{1 *}$ (D), Tiffany M. Powell-Wiley ${ }^{2,3}$, Kimberly R. Middleton ${ }^{1}$, Brenda Roberson ${ }^{1}$, Sharon Flynn ${ }^{1}$, Alyssa T. Brooks ${ }^{1}$, Narjis Kazmi ${ }^{1}$, Valerie Mitchell ${ }^{2}$, Billy Collins ${ }^{2}$, Rachel Hingst ${ }^{1}$, Lucy Swan ${ }^{1}$, Shanna Yang ${ }^{1}$, Seema Kakar ${ }^{4}$, Timothy Harlan ${ }^{4}$ and Gwenyth R. Wallen ${ }^{1}$

\begin{abstract}
Background: Cooking interventions have increased in popularity in recent years. Evaluation by meta-analyses and systematic reviews show consistent changes in dietary quality reports and cooking confidence, but not of cardiovascular (CVD) biomarkers. Interventions evaluating or reporting behavioral mechanisms as an explanatory factor for these outcomes has been sparse. Moreover, evaluations of cooking interventions among communities with health disparities or food access limitations have received little attention in the literature.

Methods: This study will occur over two phases. Phase 1 will assess acceptability among the target population of African-American adults living within an urban food desert. Phase 2 will consist of a 6-week cooking intervention delivered at a community kitchen setting. Pre and post intervention visits for clinical examinations and biomarker collection will be conducted, as well as dietary and cooking skill assessments. Primary outcomes include cooking behavior and feasibility measures. Secondary outcomes are related to dietary quality, psychosocial factors, CVD biomarkers, and food environment measures.
\end{abstract}

Discussion: This study seeks to demonstrate feasibility of a community-based cooking intervention and to provide necessary information to plan future interventions that identify cooking behavior as an outcome of participation in cooking interventions among African-American adults, especially in relation to dietary and biomarker outcomes.

Trial registration: This study was registered at ClinicalTrials.gov (NCT04305431) on March 12, 2020.

Keywords: Dietary behavior, Culinary medicine, Cooking intervention, CBPR, Feasibility study

\footnotetext{
* Correspondence: nicole.farmer@nih.gov

${ }^{1}$ National Institutes of Health, Clinical Center, Bethesda, MD, USA

Full list of author information is available at the end of the article
}

(c) The Author(s). 2020 Open Access This article is licensed under a Creative Commons Attribution 4.0 International License, which permits use, sharing, adaptation, distribution and reproduction in any medium or format, as long as you give appropriate credit to the original author(s) and the source, provide a link to the Creative Commons licence, and indicate if changes were made. The images or other third party material in this article are included in the article's Creative Commons licence, unless indicated otherwise in a credit line to the material. If material is not included in the article's Creative Commons licence and your intended use is not permitted by statutory regulation or exceeds the permitted use, you will need to obtain permission directly from the copyright holder. To view a copy of this licence, visit http://creativecommons.org/licenses/by/4.0/ The Creative Commons Public Domain Dedication waiver (http://creativecommons.org/publicdomain/zero/1.0/) applies to the data made available in this article, unless otherwise stated in a credit line to the data. 


\section{Background}

Disparities in cardiovascular disease (CVD) morbidity and mortality among African-Americans persist despite advances in risk factor identification and evidence-based management strategies [1]. Studies suggest risk factor reduction positively attenuates the mortality disparity between African-Americans and other racial/ethnic groups [1]. Closing the CVD disparity gap may require attention to more proximal causes of CVD that lower risk factors, including cardiovascular health behaviors such as dietary intake and physical activity.

Dietary behaviors consist of eating habits, food/nutrition choices, and meal preparation or cooking behavior. Among dietary behaviors, national survey data show those who frequently prepare meals at home have a better diet quality overall determined by higher vegetable intake [2] and lower daily calorie intake [3]. Observational studies show possessing a greater number of cooking skills is linked to healthier food choices [4]. However, cooking and meal preparation at home have decreased in the United States (U.S.) [5], while there has been an increase in consumption of food away from home [6]. Consequently, it is possible that the inability for Americans to meet the recommended guidelines for fruit and vegetable consumption [7] may be a public health consequence of the reduction in cooking at-home and shift to convenience foods.

The trend toward less cooking is also occurring in racial minority populations in the U.S., with AfricanAmericans reporting cooking dinner at home less than other racial/ethnic populations [8]. An updated analysis of U.S. Adult Time Use survey data showed AfricanAmerican men were the racial and gender group least likely to report engaging in meal preparation activities [9]. Our own group's NHANES (National Health and Nutrition Examination Survey) analysis of cooking frequency among African-Americans found that employment, income, and self-perceived diet quality were significant determinants of home cooking frequency [2]. Cross-sectional studies from an African-American population in a Baltimore food desert show that self-efficacy and beliefs are also contributors and are positively associated with cooking frequency and type of home cooking method $[10,11]$.

When evaluating published cooking interventions, studies among African-Americans from faith-based and community settings show successful self-report of dietary quality for improved fruit and vegetable consumption and Dietary Approaches to Stop Hypertension (DASH) score post cooking interventions [12-15]. This is in contrast to nutrition education studies that allowed participants to make their own food for the DASH diet [16]. In these studies, African-Americans were less likely as compared to
Non-Hispanic Whites to be adherent to the DASH eating plan [16]. The differing results between cooking intervention studies and free-living studies suggest an important role of cooking interventions on implementation of a cardiovascular protective dietary plan among African-Americans.

Cooking can be defined as a goal-directed behavior, where the goal is to create a meal/dish to satisfy nutrition needs or tastes for oneself or others. Two theoretical frameworks related to goal-directed behaviors that pertain to current understanding of facilitators and barriers for cooking are the social cognitive theory (SCT) and the theory of planned behavior (TPB). Both theories include individual factors related to home cooking, including self-efficacy (Fig. 1). Selfefficacy is defined as a person's belief in their capabilities to produce designated levels of performance that exert influence over events that affect their lives. It is an important construct in determining someone's motivation for a health behavior change. In terms of cooking behavior, this can transfer into self-efficacy surrounding cooking tasks, such as preparing a meal or cutting up an ingredient, to shopping for particular food items, to determining meals based on desire to change or improve one's health. These self-efficacy influences are built into cooking interventions that include aspects of personal agency, also known as food agency [17].

\section{Gaps in the literature}

Recent systematic reviews of community level cooking interventions found significant improvements in attitudes, cooking self-efficacy, and healthy dietary intake and participation in cooking interventions $[18,19]$. However, no significant outcomes are reported for cardiometabolic risk factors, including blood pressure and body mass index (BMI) within these reviews. Although methodological variation and high risk of bias from studies were identified, the findings are similar to earlier systematic reviews of cooking interventions [20]. The current literature thus leaves several questions to be addressed. If dietary intake changes and cooking efficacy changes are occurring, what is the behavioral pathway for these changes? Furthermore, what behavioral pathways may be needed or are sufficient for the expected biomarker changes to occur?

A combination of better informed food purchasing for home consumption, better choices when eating out, or more frequent home cooking may lead to dietary benefits, but ultimately may not explain needed or sufficient behavioral mechanisms. For example, practice and modeling are tenants of the SCT construct of self-efficacy. Participants may receive practice and modeling within intervention sessions 


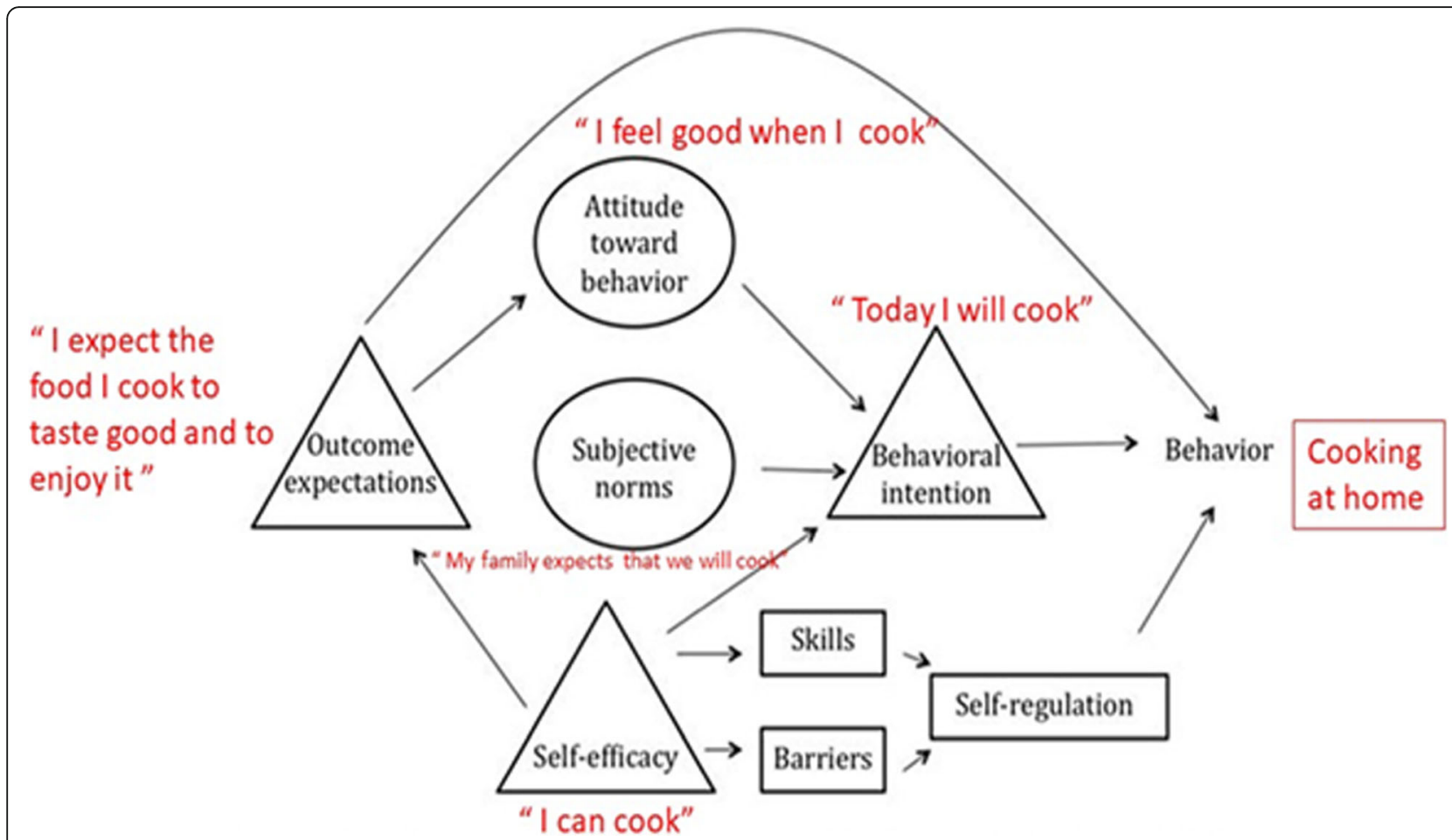

Fig. 1 Diagram of cognitive processes and constructs involved in theory of planned behavior and social cognitive theory adapted for hypothesized cognitive processes involved in home cooking. Constructs from SCT are represented by rectangles. Constructs from TPB are represented by circles. Constructs common to both are represented by triangles. Adapted from Koenings M and Arscott S, 2013

that contribute to self-efficacy, but may not have these same opportunities transfer to the home environment if home cooking behavior is not the behavior mechanism. Thus, it is conceivable that if opportunities for practice only occur within the intervention, then long-term behaviors needed for cardiometabolic change may not occur. Despite this, most cooking intervention studies only include dietary quality or health outcome variables as the primary outcomes reported [21], not cooking behavior. Furthermore, identification of cooking behavior as a mechanism is important, as cooking has the potential advantage of being more economical [22] or conferring a psychosocial benefit [23] that may be of special benefit to communities disproportionately affected by diet related chronic diseases.

The current gaps in the literature around the role of behavior may also occur because assessing behavioral mechanisms for cooking intervention outcomes can be complex. For instance, the concept of cooking skills is representative of a confluence of skills: perceptual and conceptual, mechanical, academic (knowledge of preparing raw and cooked foods), and cognitive planning skills. Additionally, for each individual, the interplay between these skills may differ based on their intrapersonal or interpersonal experiences. Lastly, the absence of a detectable effect may not result from the cooking intervention being ineffective, but rather could be a consequence of the logistical difficulties in standardizing and evaluating complex health interventions, especially in an experimental process [24]. Ideal participation and engagement level in an intervention to achieve an increase in cooking frequency is not well defined in the literature. Furthermore, it is unclear if participation and engagement level is expected to vary between individuals, or by objectively determined baseline cooking skill.

Feasibility allows for focus on real-world acceptability and implementation, while involving key stakeholders (study participants) at each stage of the exploration process [25]. A feasibility study approach also allows for process evaluations that provide a detailed understanding of complex interventions by examining their implementation, mechanism of impact, and context [26]. In relation to facilitators and barriers to cooking, understanding aspects of feasibility could help inform behavioral mechanisms that can explain cooking intervention results. The purpose of this protocol paper is to present the design and conceptualization of a cooking intervention feasibility study among African-American adults living within a food desert to evaluate home cooking behaviors pre and post a community cooking intervention. 
Based on the limited body of evidence regarding the implementation and biobehavioral outcomes of cooking interventions, the aims for the D.C. COOKS with Heart study are to:

1. Determine feasibility of delivery of cooking behavior intervention for African-American adults at risk for CVD as described by Bowen et al. (25):
a. Demand
b. Adherence to the sessions
c. Acceptability (e.g., content and delivery)
d. Practicality (e.g., mode of delivery)
e. Integration/implementation (facilitators and barriers) to home cooking

2. Determine outcomes of secondary areas of interest related to dietary quality, CVD risk factors, health behaviors, and psychosocial factors in order to gain insight into the relationship of these factors and home cooking behavior.

Table 1 shows specific primary and secondary aims for the study.

\section{Study design}

The study design uses a feasibility study model with a mixed-method research approach. The study will occur in two phases. Phase 1 will consist of focus groups with the primary aims to identify and assess current facilitators and barriers to cooking behavior in the community to inform the study. Phase 2 will consist of the cooking intervention and clinical assessment visits at baseline and at two follow up periods, 6 weeks and 12 weeks post intervention. Overall, phase 2 will have four different time points: baseline clinical visit $\left(\mathrm{TP}_{0}\right)$, cooking intervention $\left(\mathrm{TP}_{1}\right)$, second clinical visit occurring 6 weeks after the intervention concludes $\left(\mathrm{TP}_{2}\right)$, and a subsequent clinical visit occurring 12 weeks post intervention $\left(\mathrm{TP}_{3}\right)$.

Table 1 Specific study aims

\begin{tabular}{|c|c|}
\hline \multicolumn{2}{|l|}{ Phase 1} \\
\hline $\begin{array}{l}\text { Primary } \\
\text { aims }\end{array}$ & $\begin{array}{l}\text { a) Assess acceptability of the cooking intervention } \\
\text { delivery and content (recipes) } \\
\text { b) Identify facilitators and barriers to cooking frequency } \\
\text { among focus group members }\end{array}$ \\
\hline \multicolumn{2}{|l|}{ Phase 2} \\
\hline $\begin{array}{l}\text { Primary } \\
\text { aims }\end{array}$ & $\begin{array}{l}\text { a) Determine feasibility of the intervention, especially in } \\
\text { association with facilitators, or barriers to cooking }\end{array}$ \\
\hline \multirow[t]{3}{*}{$\begin{array}{l}\text { Secondary } \\
\text { aims }\end{array}$} & $\begin{array}{l}\text { a) Explore the relationship between feasibility measures } \\
\text { with intrapersonal, social and built environment factors }\end{array}$ \\
\hline & $\begin{array}{l}\text { b) Explore the relationship between feasibility measures } \\
\text { with dietary quality ( } 24 \text { hour diet recall, Mediterranean } \\
\text { Diet Score, Healthy Eating Index scores) }\end{array}$ \\
\hline & $\begin{array}{l}\text { c) Explore the relationship between feasibility measures } \\
\text { with CVD biomarkers and anthropometric measurements }\end{array}$ \\
\hline
\end{tabular}

The protocol for D.C. COOKS follows the SPIRIT checklist [27]. Table 2 shows the overall study plan and design.

\section{Study population and settings Development of D.C. COOKS with Heart}

The relevance of a study can be enhanced, and the retention of study participants improved, when community members' knowledge and experience informs the design of the intervention and dissemination of findings [28]. Community-based participatory research (CBPR) focuses on a research topic of importance to the community and ensures that intervention strategies designed using this approach are compatible with the culture and life circumstances of the target community and population being studied $[29,30]$.

A previous CBPR cardiovascular health and needs assessment of a sample population from predominantly African-American churches in wards 5, 7, and 8 was conducted through the Heart Health Study in Washington D.C. to develop a community-based behavioral weight loss intervention (Protocol: 13-H-0183; NCT 01927783 PI: Powell-Wiley). The health and needs assessment screening involved measuring cardiovascular health factors within the church-based population; and evaluated social determinants of health factors that impact weight loss. The original protocol established a community advisory board, Washington, D.C. Cardiovascular Health and Obesity Collaborative Cooking Survey (DC $\mathrm{CHOC}$ ), to consult on the planning and implementation of the assessment, as well as the interpretation and dissemination of findings from that study.

Results from the community-based cardiovascular health and needs assessment were used to inform the design and implementation of this current communitybased protocol. The current study proposal and concept were presented to DC CHOC to evaluate whether members perceived the aims of the study as relevant to the community's interest with regard to limited dietary choices and to gauge the community's interest in intervention studies which might improve dietary behaviors among African-Americas living in wards 7 and 8 . The committee provided input on intervention location and delivery, including selection of the interventionist.

Both ward 7 and ward 8 represent areas that are historically African-American, and are geographically separated from the rest of the city by the Anacostia River. While the population is diverse in economic and education levels, both communities represent significant economic and health disparities compared with the rest of the city. Both communities have the highest obesity prevalence and cardiovascular-related health events for Washington, D.C. [31], and both are considered urban food deserts. The term food desert refers to geographic 


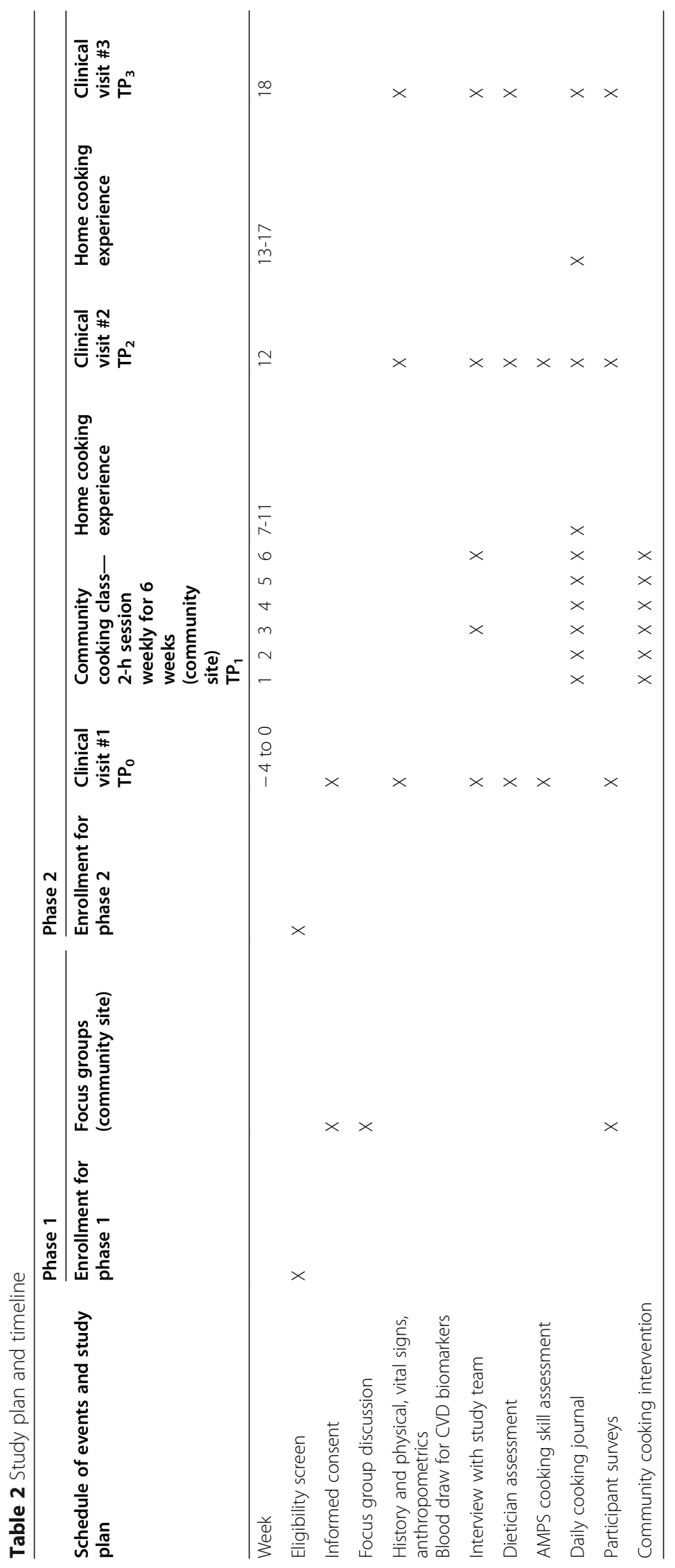


areas where most people have limited access to healthy food as defined by (1) located more than half a mile from a grocery store or supermarket or (2) low rates of car access [32]. Limited access to food is a problem that affects millions of Americans every year, and these areas tend to have concentrations of low-income and minority residents, invoking socioeconomic and racial disparities. In 2016, there were 49 grocery stores in D.C. and the average number per ward was six. However, between wards 7 and 8 , there are only three grocery stores for more than 140,000 residents [33]. The communities' location within a food desert therefore limits access to fresh foods that are most often readily used for cooking, which is a limitation that may relate to participation in cooking behavior, and food purchasing choices.

\section{Study population}

Based on the prior CBPR study, the proposed target communities for this study show a need for dietary CV risk reduction based on the risk for obesity, but also on dietary intake. In the aforementioned CBPR study, a low daily fruit and vegetable intake level of 3.2 servings per day was found compared to recommended guidelines of five per day [34]. Given the risk for CV disease, and need for improved dietary intake of fruits and vegetables, identifying ways to lower cardiovascular risk through cooking behavior within these communities may be important. Adults are the focus for this intervention and not family or child-based, because in most families adults are the nutritional decision makers [35].

Discussing health disparities requires attention and justification to how race and ethnicity are conceptualized, described, and utilized within scientific studies [36]. It is important to identify dietary quality among AfricanAmericans, because African-Americans disproportionately live in underserved areas that are subject to social, political, and policy-related pressures that determine their food environments. Thus, the selection of African-Americans in this study stems not because unhealthy diets are intrinsic to African-Americans [37, 38], but because both wards 7 and 8 are predominately African-American, 92.4\% and $92.1 \%$, respectively [39]. Therefore, most adults who are at risk for CV disease within these communities are AfricanAmerican. Moreover, within our community-based study, selection of one racial/ethnic group allows for focused insight into diet related social determinants and CVD health risks that are related to the experiences of underserved African-Americans in similar communities.

\section{Inclusion and exclusion criteria}

Participants will be eligible for this study if they are at least 18 years of age, self-identify as African-American, English-speaking, live within the designated neighborhoods of Washington, D.C. (i.e., ward 7 or 8), and self- report a risk factor for cardiovascular disease: overweight or obese, type 2 diabetes, pre-diabetes, current or recently (12 months prior) former smoker, and hyperlipidemia.

\section{Methods \\ Study settings}

Both the phase 1 focus group and phase 2 intervention sessions will be conducted within ward 7 at a location central to both Washington, D.C. communities of interest for the study, wards 7 and 8 .

The clinical setting for the study will be the National Institutes of Health (NIH) Clinical Center (CC), the U.S.'s only hospital devoted solely to clinical research. The hospital is located in Bethesda, MD, a suburb of Washington, D.C. Research participants at the NIH CC are evaluated in both outpatient and inpatient settings. For this study, an outpatient clinic utilized in the prior CBPR study will be utilized for all clinical visit appointments.

\section{Study procedures}

In phase 1 of this study, participants $(n=20)$ will take part in one of two moderated focus groups designed to explore participants' experiences with food and dietary selections and cooking behavior. Within this phase, participants will also take self-administered surveys related to cooking behavior, non-dietary health behaviors, and psychosocial factors. Open-ended (qualitative) questions for the focus groups were selected to elicit feedback regarding facilitators and barriers to cooking and perceptions about the feasibility of the intervention regarding (1) obtaining food for cooking, (2) use of cooking skills, (3) social norms around home cooking, (4) personal attitudes and knowledge, (5) preferences for recipes/food choice, and (6) preferences for intervention timing. Focus group feedback will also be used to modify the survey, if needed. Data analysis is anticipated to occur over a 1-month time period and the results of data analysis will inform the second phase. Specifically, the data captured from this phase will inform phase 2 in terms of recipe selection for intervention, local locations to source food for the intervention, anticipated concerns about intervention location, and schedule of sessions.

Phase 2 of the study consists of two parts: clinical visits, surveys, and interviews at pre $\left(\mathrm{TP}_{0}\right)$ and post intervention intervals $\left(\mathrm{TP}_{2}, \mathrm{TP}_{3}\right)$ and the cooking intervention $\left(\mathrm{TP}_{1}\right)$. We will recruit thirty $(n=30)$ participants for this phase. Phase 2 will start with a baseline visit $\left(\mathrm{TP}_{0}\right)$ prior to the intervention start. During this visit, a clinical history and physical examination, objective cooking assessment, dietician interview, and phlebotomy for CVD biomarkers will take place. Following completion of baseline visits for all participants, the 
community intervention will start with all participants who have completed the initial clinical visit. We estimate that it will take 4 weeks for all participants to complete their baseline visit. Participants will return for the second clinical visit at 6 weeks and 12 weeks post the intervention. To ensure that time of participation and days of follow up in the study are equal among participants, those who enroll and come in for their baseline visit at the earliest time point (week 4) will return for follow up visits prior to participants who completed baseline visits closer to the end of the initial time point $\left(\mathrm{T}_{0}\right)$.

\section{Cooking intervention}

The 6-week cooking intervention $\left(\mathrm{TP}_{1}\right)$ in phase 2 will be delivered by a chef who is professionally trained, licensed, and certified regarding local food safety regulations. The intervention site will be a community-based kitchen site located within ward 7 or 8. Participants will be divided into two groups of 15 . Each group will meet weekly at the kitchen site for a 90-min culinary education session. There will be no group assignment; participants may choose which group to attend, but can only attend one group session per week. The minimum number of participants required for delivery of the intervention will be one participant per session. In case of the need for an emergency medication administration for unknown food allergies, all sessions will be staffed with clinical research team members. Medical students trained in culinary medicine will also be present during each session to assist participants as needed. Table 3 provides an overview of the DC COOKS with Heart cooking intervention sessions.

\section{Intervention curriculum}

Dietary outcomes from community cooking interventions have focused on adherence to those diet patterns related to cardiovascular health, Mediterranean diet or DASH diet. The Mediterranean diet is a dietary pattern known to reduce CV disease and mortality risk [40], presumably due to promotion of higher monounsaturated fat, fruit, and vegetable consumption. Moreover, cooking

Table 3 D.C. COOKS with Heart cooking intervention

\begin{tabular}{ll}
\hline Goal & $\begin{array}{l}\text { Deliver cooking behavior intervention in a } \\
\text { community setting }\end{array}$ \\
\hline Type & In-person, chef-led instruction \\
Duration & 6-week intervention with weekly 2-h sessions \\
Structure of & $\begin{array}{l}\text { Introduction and discussion of recipes and } \\
\text { ingredients } \\
\text { sessions }\end{array}$ \\
& $\begin{array}{l}\text { Cooking of recipes in groups } \\
\text { Shared meal experience }\end{array}$ \\
Assessments & $\begin{array}{l}\text { Semi-structured interviews (phone administered) } \\
\text { Treatment fidelity } \\
\text { Behavior change taxonomy (BCT) }\end{array}$ \\
\hline
\end{tabular}

is reported as an important component of sustaining the Mediterranean diet [41]. The "Health meets Food" curriculum from the Goldring Center for Culinary Medicine (GCCM) will be used for the intervention. This longitudinal six-module curriculum is focused on the use of cooking skills to prepare foods from the Mediterranean diet. GCCM educates patients from the community in nutrition information for immediate and long-term social capital development by hands-on cooking and nutrition education [42]. The curriculum was created from prior evidence-based curricula surrounding preventive nutrition. At each session, learning objectives stem from the standard GCCM curriculum on the Mediterranean diet and nutrition education needed to start or maintain the diet. Food groups and principles of the Mediterranean diet, which include consumption of fruits and vegetables, nuts, seafood, and poultry, are highlighted in each session. The curriculum translates the Mediterranean diet for culture-specific kitchens across different socioeconomic levels [42]. In this manner, the participants are introduced to relatable food items that fall within food group categories of the Mediterranean diet. Table 4 shows a list of cooking sessions with topics, potential recipes, and study evaluation measures.

Mediterranean diet-adapted recipes in the culinary education "Health meets Food" curriculum have been utilized within a racial/ethnic and economically diverse community in the Southern U.S. In a randomized controlled trial utilizing the curriculum, a primarily AfricanAmerican (75\%) type 2 diabetic population showed a reduction in hemoglobin A1c up to 6 months post the intervention [43]; with $46 \%$ of the study population living in a food desert. The GCCM curriculum is also reflective of the principles of food agency [17, 44], in that participants prepare their ingredients to mis en place standard, work cooperatively in pairs, conversation and comparison is encouraged among pairs, and a meal is shared to engage in sensory evaluation and future meal planning. During the first session, participants will receive kitchen safety orientation provided by the chef following standard curriculum kitchen safety guidelines.

In the current protocol, recipe selection will occur in conjunction with the study team and chef interventionist along with suggestions from community members who participate in phase 1. Barriers around food sourcing that may be a result of the neighborhood food environment or location will be discussed, and when possible food sourcing for recipes will come from locations within the community.

Each intervention will consist of a discussion of a nutrition topic and of related recipes, as well as updates from prior classes including any meals/recipes made by the participants' at home. Participants will then work in groups of three to four to prepare assigned or selected 
Table 4 Cooking intervention sessions with topics, potential recipes, and study evaluation measures per session for D.C. COOKS

\begin{tabular}{|c|c|c|c|}
\hline $\begin{array}{l}\text { Session/ } \\
\text { Lesson }\end{array}$ & Topic & Potential recipes & $\begin{array}{l}\text { Study evaluation } \\
\text { measures collected }\end{array}$ \\
\hline 1 & $\begin{array}{l}\text { Mediterranean diet: Introduction to cooking } \\
\text { and reading recipes }\end{array}$ & $\begin{array}{l}\text { Salad with red wine vinaigrette and whole grain spaghetti } \\
\text { with meat and lentils }\end{array}$ & $\begin{array}{l}\text { Cooking diaries } \\
\text { Grocery receipts }\end{array}$ \\
\hline 2 & $\begin{array}{l}\text { Macronutrients: dairy, breakfast, and } \\
\text { understanding nutrition labels }\end{array}$ & $\begin{array}{l}\text { Spinach and Cheese frittata } \\
\text { Oat pancakes }\end{array}$ & $\begin{array}{l}\text { Cooking diaries } \\
\text { Grocery receipts }\end{array}$ \\
\hline 3 & Vegetables: portion sizes and lunch & One pot bean chili and tomato and cucumber salad & $\begin{array}{l}\text { Cooking diaries } \\
\text { Grocery receipts } \\
\text { Semi-structured interview }\end{array}$ \\
\hline 4 & $\begin{array}{l}\text { Legumes: good shopping habits and } \\
\text { delectable dinners }\end{array}$ & Black bean burgers with balsamic marinated mushrooms & $\begin{array}{l}\text { Cooking diaries } \\
\text { Grocery receipts }\end{array}$ \\
\hline 5 & Carbohydrates and snacks & Coconut pecan date rolls and fudgy black bean brownies & $\begin{array}{l}\text { Cooking diaries } \\
\text { Grocery receipts }\end{array}$ \\
\hline 6 & Fats and cholesterol & $\begin{array}{l}\text { Honey mustard pork tenderloin, savory braised collard } \\
\text { greens and strawberry salad with honey lime vinaigrette }\end{array}$ & $\begin{array}{l}\text { Cooking diaries } \\
\text { Grocery receipts } \\
\text { Semi-structured interview }\end{array}$ \\
\hline
\end{tabular}

recipes. During the process of cooking the recipes, the interventionist (chef) and culinary medicine, trained medical students will assist participants with instructions or techniques, if needed. Following the cooking process, a shared meal will occur in which each group will discuss what they cooked and the interventionist/students will be available for any questions. Final clean-up of the kitchen space will occur after the shared meal.

\section{Intervention assessments}

During the six intervention sessions, individualized semi-structured interviews will occur with participants at the mid-point (3 weeks) and end-point of the intervention (6 weeks). Responses to interviews will be used to help assess feasibility, and mechanisms of cooking behavior. Interviews maybe administered by phone to participants. At the conclusion of the intervention, the semi-structured interview will also include exit interview questions regarding the intervention only. Research staff will take field notes at all intervention sessions to evaluate treatment fidelity, delivery of information, and culinary education. In addition, intervention characteristics such as hands-on activity time versus lecture/listening time will be recorded for each session.

Following the principles set forth by the NIH Science of Behavior Change Program [45], interventions to change health behaviors ought to be guided by a mechanisms-of-change hypothesis. A recent review of cooking interventions by Hollywood et al. [21] found several key behavior change techniques that may contribute to dietary changes from cooking interventions: (1) information on how to perform the behavior; (2) prompt practice (prompting a person to carry out a practical task related to cooking skills or cooking behavior once or numerous times); and (3) information on the consequences of the behavior tailored to the individual [21]. As noted by identified gaps in the literature, none of the reviewed interventions measured cooking behavior as an outcome. Therefore, behavioral mechanisms for cooking behavior within cooking interventions still need to be identified. Within our study, research staff will evaluate the behavioral change techniques present in the intervention design and those presented by participants within semi-structured interviews during the cooking intervention and during post-intervention clinic follow-up visits. The rubric for the behavioral change techniques will follow those of the Coventry, Aberdeen, and London-Refined (CALO-RE) taxonomy [46] as revised by Hollywood et al [21] (Fig. 2).

\section{Study outcome measures \\ Primary outcomes}

The primary outcomes for this study are feasibility measures, home cooking behavior, and objective evaluation of cooking motor and process skills. In order to determine feasibility of the cooking intervention, we are collecting information on number of screened participants, number of participants enrolled, attendance at intervention sessions, and attrition rates. Attendance will be taken at each session. At a minimum, participants are counted as an attendee when one full intervention session has been completed. Completers of the intervention will be defined as participants who complete all six intervention sessions. Dropouts for the study will be defined as those who attend the first clinical visit, but do not attend any intervention session by the date of the final session. Attrition rates will be determined from the number of participants who stopped participating divided by the average number of participants at each time point, and over the total study period (see Fig. 4 for time points). We anticipate that our attrition rates will be at or below 33\%, which is the reported attrition rate for this target population based on members of the research team's experience recruiting from wards 7 and 8 [34]. 


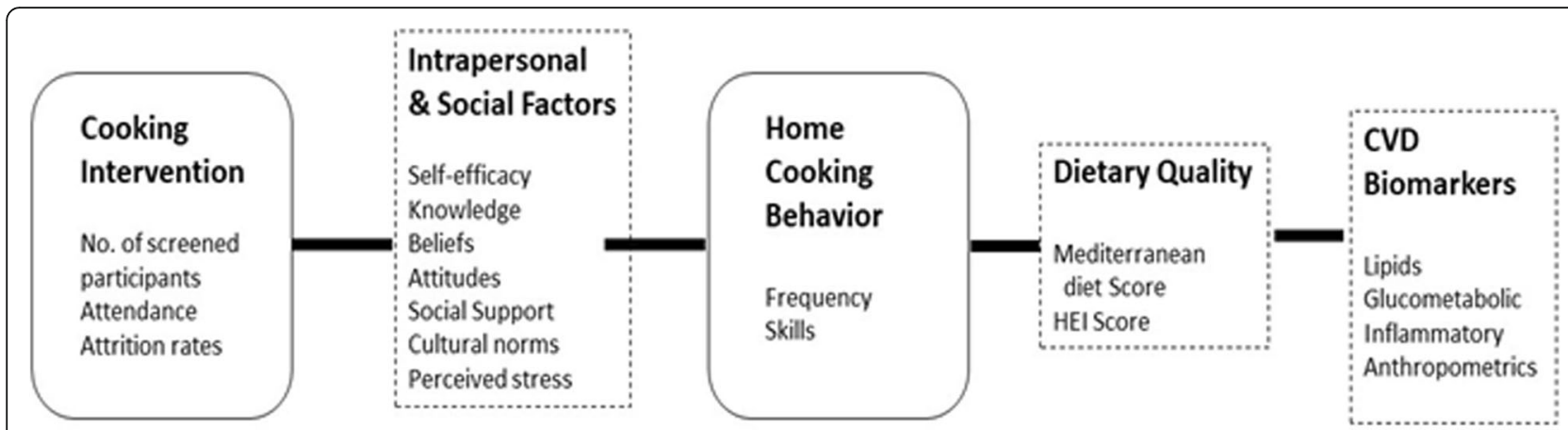

Potential mediators or confounders: demographics, lifestyle behaviors (sleep, physical activity), built environment, self-rated health

Fig. 2 Conceptual model of potential relationships between primary and secondary outcomes

Feasibility success will be achieved if the majority of participants $(>50 \%)$ reach $50 \%$ participation ( $3 / 6$ sessions). This is based on two reports in the literature of cooking intervention studies among African-Americans in which this attendance metric was met [13, 15]. Information collected on feasibility and cooking behavior and skills will allow for appropriate sample size calculations for future studies.

Cooking behavior will be determined through multiple variables to provide a convergence of data. Using paper cooking diaries, participants will be instructed to start daily recording of home cooking following the initial clinical visit and will stop after the last visit, for a total collection time of 18 weeks. The design for the cooking diaries is fixed-schedule (once per day). Each daily entry will consist of marking the frequency of cooking at home, as well as commenting on enjoyment, interest [47], and perception of cooking skills.

Figure 3 illustrates the questions and answer choices for each cooking diary entry. At each clinical visit (TP0, TP2, TP3), additional measures of cooking behavior will be collected. Home cooking frequency, family meal behavior, and frequency of purchasing foods outside of the home over the prior 7 days will be determined using standardized national survey questions [48, 49]. Self-efficacy for cooking and food agency will be determined from validated survey tools $[14,44]$. Psychosocial determinants of cooking will be assessed with a survey tool pre-tested within the target community by our team [50].

As stated earlier, a current gap in the literature is whether participation and engagement level in cooking behavior is expected to vary by participant's baseline cooking skill. To our knowledge, assessment of baseline cooking skill has not been described in published cooking intervention studies as a participant characteristic. Assessment of Motor and Process Skills (AMPS) is a kitchen performance assessment test delivered by occupational therapists to assess an individual's performance skills (motor and process). For each task, motor and process skills are evaluated. Motor skills involve hand movement and coordination. Process skills involve the actions used to organize and adapt behavior over time in order to complete a task. The AMPS test will be administered by trained occupational therapists in the Department of Rehabilitation kitchen at the NIH Clinical Center at baseline and the 6-week clinical visit. Analysis of AMPS will be done with a computerized scoring system where the correction for the score is based on skilled item difficulty, task challenge, and rater severity [51]. Because the test encourages participants to choose familiar tasks, it is participant centered. Additionally, AMPS has been validated for use in an AfricanAmerican adult population [52].

\section{Secondary outcomes}

Health behaviors are often socially patterned [67]. Moreover, dietary behaviors are likely influenced by tradition and a shared history that is passed from generations which then determine intrapersonal (taste preferences), interpersonal (cultural and familial paradigms), and community set norms [67]. Therefore, secondary outcomes measured are related to psychosocial factors, the neighborhood food environment, dietary intake, and health behaviors, such as physical activity and sleep quality. Figure 2 illustrates our conceptual model of potential relationships between primary and secondary outcomes. Table 5 provides a list of primary and secondary study measures with instrument type and description of measurement.

All secondary outcomes will be obtained through selfadministered questionnaires, except for dietary intake. Dietary intake will be measured using a 24-h recall of food and drink intake collected by the nutrition team from the NIH Clinical Center Nutrition Department. 


\section{Cooking Frequency}

Circle the meals that you prepared/cooked

breakfast lunch dinner other

\section{Household Involvement}

Circle the person you prepared/cooked

family friend other

with if anyone

Circle the person you prepared/cooked for

family friend other

if anyone

\section{Enjoyment}

Please rate on a scale of 0 to 10 (zero as not at all, 10 as very much),

how much did you enjoy preparing/cooking today?

Please share/describe any thoughts on this experience

open text field

Did you find cooking interesting or fun today?

yes no

\section{Challenge}

Please rate on a scale of 0 to 10 , how much was preparing/

cooking challenging today?

Please share/describe any thoughts on this experience

open text field

\section{Cooking as required task}
Did you have to cook today?
yes no
Did you want to cook today?
yes no

Fig. 3 Cooking diary components by topics. Participants will be instructed to respond for each day of the week

During the interview, the nutrition staff member will ascertain the content and quantity of dietary intake, as well as inclusion of home cooked versus purchased foods. This will be done to determine baseline and post intervention diet quality of home cooked food, not just general diet quality. Data from the 24-h recall will also provide information regarding dietary patterns. Study staff (registered dieticians) will analyze the food records using Nutrition Data System for Research (NDS-R) software for energy, protein, carbohydrate, fat, alcohol, caffeine, and micronutrient intake [68]. A composite diet quality score ranging from 0 to 100 will be calculated using the Healthy Eating Index, HEI-2015 (https://epi. grants.cancer.gov/hei/) [65]. Calculated HEI score 
Table 5 Table of study measures with instrument type and description of measurement

\begin{tabular}{ll}
\hline Measurement & Type of instrument(s) \\
\hline Primary outcomes & \\
Facilitators and barriers to cooking \\
Cooking diaries & Daily self-administered data collection \\
Cooking self-efficacy scale & Self-administered 7 item instrument \\
(CSES) $[14,53]$ & \\
Psychosocial factors related & Self-administered 32 questions (61 items) D. \\
to cooking [50] & CHOC Cooking Survey \\
& \\
& \\
Food agency [17] & Self-administered 28 item Cooking and Food \\
& Provisioning Action Scale (CAFPAS)
\end{tabular}

Cooking skills [51]

Assessment of Motor and Process Skills (AMPS)

Feasibility measures Attrition

Attendance

Participant burden

Treatment fidelity [54]

Health promoting Lifestyle Profile II (HPLP-II) [57]

Perceived stress [58]

\section{Neighborhood factors}

MESA Neighborhood Perception of Healthy Food Availability Scale [59]

Attendance record at each intervention session using study log

Observations of research team and participant feedback

Self-administered shortened 3 item scale (the original being 6-item).

\section{Description}

Daily responses to cooking frequency questions will be used

CSES assesses the degree of confidence in performing basic cooking activities on a 5-point Likert scale

D. C. CHOC is a self-administered 32 question (61 items) survey to assess psychosocial determinants and developmental exposure to cooking as well as confidence for certain cooking techniques and food shopping. It will also capture cooking frequency over the last 7 days.

CAFPAS is a 28 item instrument with 3 sub-scales ( food selfefficacy -13 items, food attitude-10 items, perceived influence of non-food barriers on provisioning -5 items). The scale has undergone face and construct validity and reliability testing, with Cronbach's alpha $=0.7$

AMPS is a kitchen performance assessment test delivered by occupational therapists to assess an individual's performance skills. The AMPS will be conducted at baseline and 6-week clinic visit.

Number of participants that complete the study

Attrition will be based on the number of participants at the start and remaining at the end of the study, as well as at each time point. Attrition rates will be determined from the number of participants who stopped participating divided by the average number of participants at each time point, and over the total study period

Attendance will be taken at each intervention session and rates will be analyzed to determine the desired dosage (how much, how often and at what interval) for each participant by their characteristics

Participant burden will be determined by data collection assessments, research team's perception of participants' understanding of questions and data collection methods, and if participants respond with missing or unusable data. The study team will also assess if participants have enough time and capacity to complete data collection procedures.

Treatment fidelity assessment grid will be used to determine implementation of the intervention. Cooking diaries and interviews will also be used as a measure of implementation/ intervention fidelity.

SNI is a self-administered 12-item instrument that assesses participation in 12 types of social relationships. There are three measures within the SNI: number of high-contact roles (network diversity), number of people in social network, and number of embedded networks.

The HPLP-II is a self-administered 52-item instrument that measures the frequency of self-reported healthy behaviors. It consists of 6 subscales: physical activity, spiritual growth, health responsibility, interpersonal relations, nutrition, and stress management (including sleep quality).

PSS measures an individual's perceptions about the nature of events and their relationship to coping resources of that individual. This 10 item tool uses a 5 point Likert scale for each item.
This scale is used to calculate perceived healthy food availability in the neighborhood, which is defined as within a 20-min walk or one mile distance from the individual's home. 
Table 5 Table of study measures with instrument type and description of measurement (Continued)

\section{Measurement}

Perception of

Neighborhood Food Retail

Outlets [60]

Neighborhood satisfaction Single question with 5 answer choices [61]

Food purchasing practices Grocery receipts

[61]

Food purchasing practices

Food Away from Home frequency

Self-rated health [62]

Sleep quality assessment [63]

Physical activity [64]

HEI \& Mediterranean diet adherence screener $[65,66]$ (IPAQ)-Short form

24-h food frequency questionnaire

Self-administered 9 item questionnaire

\section{Description}

This tool consists of 9 items and tests types of retail outlets available within the neighborhood, which is defined as within a 20-min walk or one mile distance from the individual's home.

Neighborhood satisfaction will be measured with the question, "All things considered, would you say you are very satisfied, satisfied, dissatisfied, very dissatisfied, or neutral - neither satisfied nor dissatisfied with your neighborhood as a place to live?"

Grocery receipts will be collected at intervention sessions and follow up CC visits, to assess overall dietary quality and utilization of food store type

Food purchasing questionnaire measures frequency of major food shopping with 11 different types of store options. It also inquires about mode of transportation for that major food shopping trip.

Food away from home will be assessed by one question from CD-NHANES-DBQ, 2015 (During the past 7 days, how many meals did you get that were prepared away from home in places such as restaurants, fast food places, food stands, grocery stores, or from vending machines?), whereas the meal could mean breakfast, lunch or dinner.

Self-rated health is assessed through one question and it measures the general health state or change in state which could be associated with outcomes of interest

PSOI is a 9-item self-administered measure that assesses the quality and patterns of sleep. PSQI has seven subscales and altogether they create a total score of sleep quality.

International Physical Activity Questionnaire 
assesses the extent to which a participant follows the 2015-2020 Dietary Guidelines for Americans (DGA). Because the HEI-2015 aggregates dietary components, it provides a hypothesis-oriented approach to determine participants' dietary pattern. The Mediterranean Diet score will be determined by the Mediterranean Diet Adherence Screener (MEDAS) [66].

\section{Recruitment and retention plan}

For phase 1, focus groups will be completed prior to the beginning of phase 2 . Recruitment will utilize participants from the community-based participatory research study (NCT01927783) for the focus groups, as well as adults associated with collaboration sites of the DC CHOC. Initial contact will occur through phone contact with identified eligible participants. Likewise, email announcements will be sent to collaboration sites. Recruitment of focus group participants will use a purposive snowballing sample of African-American adults (age > 18) who live in wards 7 or 8 in Washington D.C. However, we will aim for the study recruitment to lead to variation in demographic subgroups. Identified participants may or may not be currently enrolled in or participated previously in a community cooking education program/class. At the time of phone contact with potential participants, study staff will ask screening eligibility questions regarding self-reported risk for cardiovascular disease. If potential participants are deemed eligible based on aforementioned inclusion/exclusion criteria, they will be invited to participate in a focus group.

For phase 2, similar strategies for identifying participants will be used as in phase 1 . At the time of contact with potential participants, study staff will ask screening eligibility questions regarding self-reported risk for cardiovascular disease. If potential participants are deemed eligible based on aforementioned inclusion/exclusion criteria, they will be invited to schedule a baseline visit. Signing of informed consent document for the protocol will be obtained at clinical visit \#1 $\left(\mathrm{TP}_{0}\right)$ after discussion and explanation of study requirements with a member of the research study team. Eligible study participants will have consent document mailed to them to review prior to clinical visit \#1. The planned flow and steps of data collection within phase 2 relevant to expected recruitment and retention are illustrated in Fig. 4.

Retention efforts focused on participant engagement include reminder phone calls to participants on a weekly basis during the intervention and 1 week and 1 day prior to all clinical visit appointments. Participants will be discontinued from the study if any of the following should occur: participant requests to be removed from the study, participant does not participate in the initial clinical visit, or participant is unable to participate in the intervention component.

\section{Analysis Qualitative data}

A phenomenological methodological approach will be undertaken in both phase 1 and 2 to understand participants' lived experiences with cooking at home within the context of their neighborhood and household. Phase 1 will focus on gathering an understanding of nonintervention based conditions. While in phase 2, we will gather an understanding of the participation experience within the specific setting of the cooking intervention. In both phases, qualitative data collection and analysis will use the four criteria that comprise the rigorous standards for qualitative research: credibility, dependability, confirmability, and transferability [69]. Credibility will be maintained by the independent analyses of our transcribed interview data to ensure that our theme categories cover all relevant data. Credibility will be further strengthened through the consensus-building process, which will ensure that similarities within and differences in our theme categories are thoroughly evaluated. Dependability will be established by maintaining facilitator consistency through data collection and through data analysis by our team. Confirmability will be supported by developing an electronic, thematic database and through the preservation of interviewer perceptions via interview/focus group field notes. Our diverse study team, comprising individuals with various clinical and research-related expertise with members of the community, will support the criteria of transferability.

In phase 1, the sources for qualitative data are the transcripts and research team field notes from the focus groups. In phase 2, the source of the qualitative data will come from semi-structured interviews conducted at all study time points. Analysis in both phases will use the de-identified verbatim transcription of audiotapes and field notes written by research team members. Members of the research team will develop a codebook based on themes from the interviews. Each thematic code will be accompanied by an operational definition that will allow for clarity and consistency in the coding process. A team of coders will independently review all transcripts. Discordant coding will be discussed until consensus among the coding team is achieved. Once the iterative process of consensus building is complete, an intramural expert in qualitative methodology will validate the final themes and coding. After data are coded, NVivo will be utilized for data management.

\section{Quantitative data}

\section{Sample size justification}

For phase 2, sample size justification was done in accordance with Billingham et al. [70]. From the literature for published cooking interventions, intervention group size ranges from 12 to 30 participants [13-15, 43]. We therefore selected a sample size of 30 participants for phase 2 . 
Inclusion
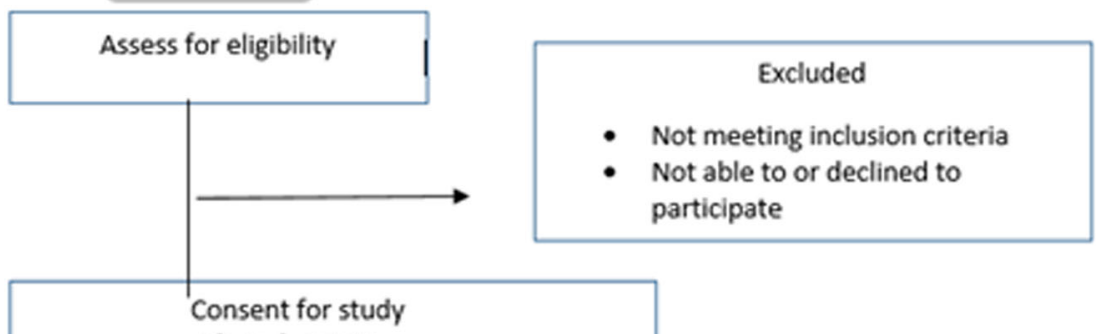

Clinical visit \#1:

- Blood pressure, weight, BMI*

- Anthropometric measurements *

- Medical and social history *

- Laboratory draw for CVD related biomarkers *

- Dietician interview -24 hour recall *

- Interview and surveys for outcome measures *

- OT Cooking Skills Assessment ^

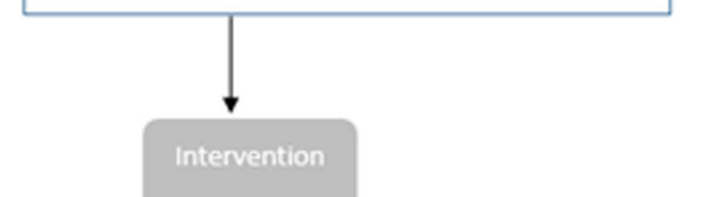

intervention

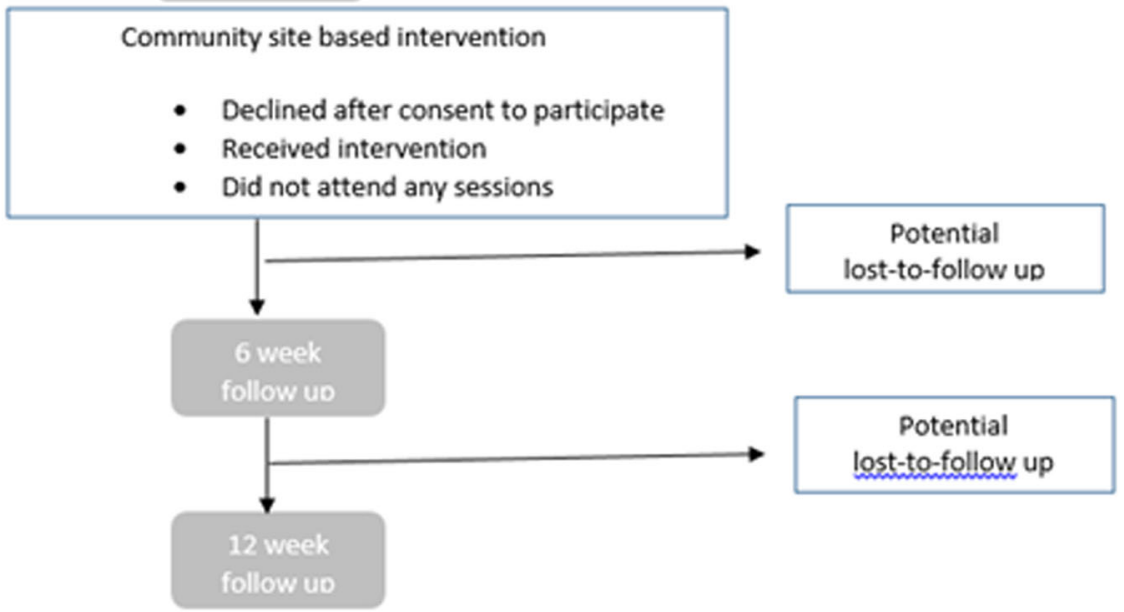

*Repeated at both 6 and 12 week follow up. ^ Repeated at 6 week follow up only.

Fig. 4 The planned flow and steps of data collection within phase 2 relevant to expected recruitment and retention

We also based the sample size on expected attrition. From our previous community study (NCT 0192778 [34];), we anticipate a $33 \%$ attrition rate for the first clinical visit. Therefore, in order to reach 30 participants, we plan to screen 45 participants.

\section{Data analysis}

Baseline demographic and clinical data will be analyzed using descriptive statistics. Categorical data will be presented as frequencies and percentages, while continuous data will be presented as means and standard deviations. Patterns of missing data will be examined thoroughly to assess whether any questions were systematically skipped by all participants or any sub-group of participants.

All continuous variables of interest for quantitative data analysis will have baseline evaluation done to check for outliers and normal distribution. Data determined to 
not have normal distribution will be expressed as medians with interquartile ranges. If the assumptions of normality are not violated, we will proceed with parametric testing. If the assumptions of normality are violated, non-parametric tests will be utilized. For quantitative analyses, statistical significance will be considered at two-tailed, $p<0.05$. Statistical analysis will be conducted in available statistical software (for example SAS, SPSS). Inferential statistics will be conducted for the purposes of exploration, but without placement of conclusions on the effectiveness of the intervention. Relationships between variables will be examined with bivariate correlation coefficients (for two continuous variables), Chi-squares (for two categorical variables), and $t$ tests. Analysis of variance (ANOVA) (for one categorical and one continuous variable) will be conducted to explore sub-group analysis. Linear or logistic regression analyses, with multivariate analysis when applicable, will also be pursued to examine determinant models of cooking behaviors or feasibility measures.

Phase 2 consists of four time points. Group level analyses will be conducted with reporting and comparison of group means at each time point. Within-person analysis using repeated measures will also be done to compare within person changes across time points, including repeated measures ANOVA and $t$ tests where applicable.

For the survey responses related to the primary outcomes, facilitators, and barriers to cooking (DC CHOC, CAFPS, CES), question items deemed as positively related to facilitating cooking will have a sum of scores calculated and then divided by total number of respondents. Survey items deemed as negatively related to cooking (barriers) will be reverse scored to determine mean scores. Within DC CHOC Survey, responses for cooking perception will be dichotomized as agreement with cooking if response is $\geq 5$, as reported in Wolfson 2016 [71].

From the cooking diaries, daily cooking frequency amounts will be aggregated into cooking frequency for each week of the study, the average at each time point of phase 2 for each participant. Cooking frequency at each time point will also be dichotomized based on the average cooking frequency per week of 10.8 out of 21 possible meals, as reported in a national survey of U.S. adults [71].

\section{Data collection and management}

All analyzed data will have unique, study-specific numerical identifiers so that research data can be attributed to an individual human subject participant. Data obtained during the conduct of the protocol will be kept in secure password-protected network drives or in approved alternative sites that comply with NIH security standards. This includes recorded participant interviews. The principal investigator and associate investigators, research nurses, and/or contracted data managers will assist with the data management efforts.

Survey measures will be collected electronically on clinic tablets used by participants on clinical visit days. Laboratory data and clinical examination data will be collected using the Clinical Center electronic health record known as Clinical Research Information System (CRIS). Cooking diary data will be completed by paper format and then entered electronically and managed using REDCap electronic data capture tools hosted within the Biomedical Translational Research Information System (BTRIS) at the NIH. REDCap (Research Electronic Data Capture) is a secure, web-based application designed to support data capture for research studies [72]. REDCap was developed and is licensed by Vanderbilt University. All use of REDCap will occur in compliance with NIH security standard. Participants without a personal electronic device will have paper cooking diaries provided for mail return to the research team in pre-stamped, self-addressed envelopes. The diaries will then be entered using a double-data entry method into a secure data management system.

Data from enrolled subjects will be stored until they are no longer deemed of scientific value or if a subject withdraws consent for their continued use, at which time they will be destroyed. Should we become aware that a major breach in our plan for tracking and storage of data has occurred, the Internal Review Board (IRB) will be notified. Each NIH protocol undergoes a yearly departmental, independent audit in addition to yearly continuing reviews by the IRB.

Data management committees (DMC) are not usually warranted in early studies such as pilot/feasibility studies, but formal monitoring groups may be useful for certain types of early clinical studies [73]. For this protocol, a study monitoring group consisting of study investigators will be formed and meet regularly throughout the study time frame. Safety monitoring will occur following standard procedures within the NIH. These procedures include reporting of adverse events related to participation in the study. Adverse events will be reported to the principal investigator (PI) with recommendations and follow up, as well as documentation in the clinical electronic patient record. Adverse events will focus on ones related to the intervention and clinical visits where the $\mathrm{NIH}$ is involved either directly or indirectly by recommending certain interventions. Adverse events associated with the cooking intervention will be monitored by the PI, serving as safety officer, and will be reported to the IRB as appropriate and as part of the annual report.

\section{Evaluation of risks/discomforts and benefits ratio}

This protocol will assess feasibility of administering a cooking behavior intervention in a community at risk 
for CVD within a food desert. Participation in phase 2 may confer a health-behavior, nutrition education based benefit to participants. However, it is possible that there may be no direct benefit. It is also possible that in both phase 1 and phase 2, answering survey questions may cause discomfort to participants. Furthermore, in phase 2 phlebotomy for laboratory study collection may lead to discomfort at the puncture site. If this should occur, every effort will be made to address and minimize participant discomfort.

\section{Compensation}

Participants will be compensated for research-related discomfort and inconveniences in accordance with $\mathrm{NIH}$ guidelines. If participants are unable to finish the study, they will be paid only for those parts completed.

\section{Dissemination of results}

This protocol was developed in collaboration with the DC CHOC community advisory board. Therefore, results of the study germane to the primary and secondary outcomes as well as results determined to be related to stated dietary behavior concerns voiced by the committee, will be presented at the DC CHOC committee meetings. Publications related to baseline data and primary outcomes will be disseminated to study participants once published.

Since the protocol is federally funded, any manuscript to be published in a peer-reviewed journal will be submitted to PubMed Central or public access upon acceptance for publication.

\section{Study strengths and limitations}

A key strength of our study is the mixed methods design, with a sequential approach in allowing the qualitative data from phase 1 inform phase 2 , and a simultaneous approach in phase 2 using both quantitative and qualitative data. Mixed methods studies are noted as important for understanding health disparities [74] and health behaviors [75]. Another strength is the use of an objective cooking skill assessment and use of over lapping measures of behavior (the primary outcome). The study's other strength is community recruitment and involvement which can help to create pragmatic results relevant to members of the community. Despite its strengths, there are some limitations to consider. Reliance on a previously identified group of participants for recruitment may limit our external validity to other members of the community who have not previously participated in community research. The use of self-reported data for secondary outcome measures that can be influenced by biases such as recall or social desirability, serves as an additional limitation.

\section{Conclusions}

With dietary guidelines focusing on foods that require cooking and preparation, such as fruits and vegetables, it is important to identify dietary behaviors which may promote adherence to current guidelines. Furthermore, for communities disproportionately affected by dietrelated chronic diseases, identifying dietary behaviors that may offer chronic disease prevention through better guideline adherence is significant. This feasibility study seeks to address a critical gap in our understanding of how cooking interventions may lead to cooking behavioral changes and what factors (intrapersonal or interpersonal) may facilitate or impede needed behaviors at home in order to follow cardiovascular preventive diets.

\section{Trial status}

This study was approved by the NIH Intramural Institutional Review Board in February of 2020 (NCT 04305431). We expect to begin recruitment in late Summer or early Fall of 2020.

\section{Supplementary information}

Supplementary information accompanies this paper at https://doi.org/10. 1186/s40814-020-00697-9.

Additional file 1. SPIRIT 2013 Checklist: recommended items to address in a clinical trial protocol and related documents.

\begin{abstract}
Abbreviations
A1C: Glycated hemoglobin; AA: African-American; AMPS: Assessment of Motor and Process Skills; ANOVA: Analysis of variance; Apo: Apolipoprotein; BMI: Body mass index; BP(s): Blood pressure; CAPFS: Cooking and Food Provisioning Action Scale; CBC: Complete blood count; CBPR: Communitybased participatory research; CC: Clinical Center; CALO-RE: Coventry, Aberdeen, and London-Refined; CRIS: Clinical Research Information System; CSES: Cooking Self-efficacy Scale; CVD: Cardiovascular disease; DASH: Dietary approaches to stop hypertension; DC CHOC CS: DC Cardiovascular Health and Obesity Collaborative Cooking Survey; DGA: Dietary Guidelines for Americans; DMC: Data Management Committee; FDA: Food and Drug Administration; HEl: Health Eating Index; HHS: Health and Human Services; HPLP-II: Health Promoting Lifestyle Profile-Il; hs-CRP: High-resolution, Creactive protein; IL-6: Interleukin 6; IPAQ-SF: International Physical Activity Questionnaire-Short Form; IRB: Internal Review Board; MEDAS: Mediterranean Diet Adherence Score; NDS-R: Nutrition Data System for Research; $\mathrm{NIH}$ : National Institutes of Health; NIHCC: National Institutes of Health Clinical Center; OT: Occupational therapist; PBC: Perceived behavioral control; PI: Principal investigator; PII: Personally; PQSI: Pittsburgh Sleep Quality Assessment; PSS: Perceived Stress Scale; REDCap: Research Electronic Data Capture; SCT: Social cognitive theory; SNI: Social Network Index; TP: Time point; TPB: Theory of planned behavior
\end{abstract}

\section{Acknowledgements}

Not applicable.

\section{Authors' contributions}

Conceptualization, NF, GW, and TPW; methodology, NF, GW, TPW, AB, SF, NK, KM, BC, VM, BR, RH, LS, SY, SK, TH; supervision, GW and TPW; writing-original draft, NF, GW, KM, NK, and TPW; writing-review and editing NF, GW, TPW, AB, SF, NK, KM, BC, VM, BR, RH, LS, SY, SK, TH. All authors read and approved the final manuscript. 


\section{Funding}

This study was funded by Intramural funding from the Clinical Center at the National Institutes of Health, the Division of Intramural Research of the National Heart, Lung, and Blood Institute and the Intramural Research Program of the National Institute on Minority Health and Health Disparities.

\section{Availability of data and materials}

Not applicable.

\section{Ethics approval and consent to participate}

All procedures performed in studies involving human participants were in accordance with the ethical standards of the institutional and/or national research committee and with the 1964 Helsinki declaration and its later amendments or comparable ethical standards. This study was approved by the Intramural NIH IRB.

\section{Consent for publication}

Not applicable.

\section{Competing interests}

T.H. reports role as President, Culinary Medicine Specialist Board (501c3 nonprofit registered in Virginia). All other authors have no competing interests to report.

\section{Author details \\ ${ }^{1}$ National Institutes of Health, Clinical Center, Bethesda, MD, USA. ${ }^{2}$ Social Determinants of Obesity and Cardiovascular Risk Laboratory, Cardiovascular Branch, Division of Intramural Research, National Heart, Lung, and Blood Institute, National Institutes of Health, Bethesda, MD, USA. ${ }^{3}$ Intramural Research Program, National Institute on Minority Health and Health Disparities, Bethesda, MD, USA. ${ }^{4}$ George Washington University School of Health Sciences, Washington, DC, USA.}

Received: 23 April 2020 Accepted: 29 September 2020

Published online: 19 October 2020

\section{References}

1. Carnethon MR, Pu J, Howard G, Albert MA, Anderson CAM, Bertoni AG, et al. Cardiovascular Health in African Americans: A Scientific Statement From the American Heart Association. Circulation. 2017;136(21):e393-423.

2. Farmer N, Wallen GR, Yang L, Middleton KR, Kazmi N, Powell-Wiley TM. Household cooking frequency of dinner among non-Hispanic Black adults is associated with income and employment, perceived diet quality and varied objective diet quality, HEI (Healthy Eating Index): NHANES Analysis 20072010. Nutrients. 2019;11(9):2057.

3. Wolfson JA, Bleich SN. Is cooking at home associated with better diet quality or weight-loss intention? Public Health Nutr. 2015;18(8):1397-406.

4. McGowan L, Caraher M, Raats M, Lavelle F, Hollywood L, McDowell D, et al. Domestic cooking and food skills: A review. Crit Rev Food Sci Nutr. 2017; 57(11):2412-31.

5. Smith LP, Ng SW, Popkin BM. Trends in US home food preparation and consumption: analysis of national nutrition surveys and time use studies from 1965-1966 to 2007-2008. Nutr J. 2013;12(1):45.

6. Saksena MJ, Okrent AM, Anekwe TD, Cho C, Dicken C, Effland A, et al. America's eating habits: food away from home. Washington, DC: United States Department of Agriculture, Economic Research Service; 2018.

7. Mozaffarian D. Dietary and policy priorities for cardiovascular disease, diabetes, and obesity: a comprehensive review. Circulation. 2016;133(2): $187-225$.

8. Virudachalam S, Long JA, Harhay MO, Polsky DE, Feudtner C. Prevalence and patterns of cooking dinner at home in the USA: National Health and Nutrition Examination Survey (NHANES) 2007-2008. Public Health Nutr. 2014;17(5):1022-30.

9. Taillie LS. Who's cooking? Trends in US home food preparation by gender, education, and race/ethnicity from 2003 to 2016. Nutr J. 2018;17(1):41.

10. Garcia MT, Sato PM, Trude ACB, Eckmann T, Steeves ETA, Hurley KM, et al. Factors associated with home meal preparation and fast-food sources use among low-income urban African American adults. Ecol Food Nutr. 2018; 57(1):13-31.

11. Henry JL, Trude ACB, Surkan PJ, Anderson Steeves E, Hopkins LC, Gittelsohn J. Psychosocial determinants of food acquisition and preparation in low- income, Urban African American households. Health Educ Behav. 2018;45(6): 898-907.

12. Allicock M, Campbell MK, Valle CG, Carr C, Resnicow K, Gizlice Z. Evaluating the dissemination of body \& soul, an evidence-based fruit and vegetable intake intervention: challenges for dissemination and implementation research. J Nutr Educ Behav. 2012;44(6):530-8.

13. Brewer LC, Balls-Berry JE, Dean P, Lackore K, Jenkins S, Hayes SN. Fostering African-American improvement in total health (FAITH!): an application of the American Heart Association's Life's Simple $7^{\mathrm{TM}}$ among Midwestern AfricanAmericans. J Racial Ethn Health Disparities. 2017;4(2):269-81.

14. Condrasky MD, Baruth M, Wilcox S, Carter C, Jordan JF. Cooks training for faith, activity, and nutrition project with AME churches in SC. Eval Program Plann. 2013:37:43-9.

15. Whitt-Glover MC, Hunter JC, Foy CG, Quandt SA, Vitolins MZ, Leng I, et al. Translating the dietary approaches to stop hypertension (DASH) diet for use in underresourced, urban African American communities, 2010. Prev Chronic Dis. 2013:10:120088.

16. Epstein DE, Sherwood A, Smith PJ, Craighead L, Caccia C, Lin P-H, et al. Determinants and consequences of adherence to the dietary approaches to stop hypertension diet in African-American and white adults with high blood pressure: results from the ENCORE trial. J Acad Nutr Diet. 2012; 112(11):1763-73

17. Lahne J, Wolfson JA, Trubek A. Development of the cooking and food provisioning action scale (CAFPAS): a new measurement tool for individual cooking practice. Food Qual Prefer. 2017;62:96-105.

18. Hasan B, Thompson WG, Almasri J, Wang Z, Lakis S, Prokop $L$, et al. The effect of culinary interventions (cooking classes) on dietary intake and behavioral change: a systematic review and evidence map. BMC Nutr. 2019; 5(1):29.

19. Reicks M, Kocher M, Reeder J. Impact of cooking and home food preparation interventions among adults: a systematic review (2011-2016). J Nutr Educ Behav. 2018;50(2):148-72.e1.

20. Reicks M, Trofholz AC, Stang JS, Laska MN. Impact of cooking and home food preparation interventions among adults: outcomes and implications for future programs. J Nutr Educ Behav. 2014;46(4):259-76.

21. Hollywood L, Surgenor D, Reicks M, McGowan L, Lavelle F, Spence M, et al. Critical review of behaviour change techniques applied in intervention studies to improve cooking skills and food skills among adults. Crit Rev Food Sci Nutr. 2018;58(17):2882-95.

22. Tiwari A, Aggarwal A, Tang W, Drewnowski A. Cooking at home: a strategy to comply with U.S. dietary guidelines at no extra cost. Am J Prev Med. 2017;52(5):616-24.

23. Farmer N, Touchton-Leonard K, Ross A. Psychosocial benefits of cooking interventions: a systematic review. Health Educ Behav. 2017;45(2):167-80.

24. Medical Research Council. Developing and evaluating complex interventions. 2009. Available from: https://mrc.ukri.org/documents/pdf/ complex-interventions-guidance/.

25. Bowen DJ, Kreuter M, Spring B, Cofta-Woerpel L, Linnan L, Weiner D, et al. How we design feasibility studies. Am J Prev Med. 2009;36(5):452-7.

26. Moore GF, Audrey S, Barker M, Bond L, Bonell C, Hardeman W, et al. Process evaluation of complex interventions: Medical Research Council guidance. bmj. 2015;350:h1258

27. Chan AW, Tetzlaff JM, Altman DG, et al. SPIRIT 2013 statement: defining standard protocol items for clinical trials. Ann Intern Med. 2013;158:200-7.

28. Cohen A, Lopez A, Malloy N, Morello-Frosch R. Our environment, our health: a community-based participatory environmental health survey in Richmond, California. Health Educ Behav. 2012;39(2):198-209.

29. Benjamin EJ, Smith SC, Cooper RS, Hill MN, Luepker RV. Task force\# 1-magnitude of the prevention problem: opportunities and challenges. J Am Coll Cardiol. 2002;40(4):588-603.

30. Ammerman A, Corbie-Smith G, St. George DMM, Washington C, Weathers B, Jackson-Christian B. Research expectations among African American church leaders in the PRAISE! project: a randomized trial guided by communitybased participatory research. Am J Public Health. 2003;93(10):1720-7.

31. US Department of Health and Human Services (DHHS). Obesity in the District of Columbia. 2010.

32. USDA Economic Research Service. 2017. Available from https://www.ers usda.gov/data-products/food-access-research-atlas/documentation/.

33. D.C. Hunger Solutions. Closing the Grocery Store Gap in the Nation's Capital. 2017. https://www.dchunger.org/wp-content/uploads/2018/11/ dchs-closing-grocery-store-gap-report.pdf. 
34. Yingling LR, Mitchell V, Ayers CR, Peters-Lawrence M, Wallen GR, Brooks AT, et al Adherence with physical activity monitoring wearable devices in a communitybased population: observations from the Washington, DC, Cardiovascular Health and Needs Assessment. Transl Behav Med. 2017;7(4):719-30.

35. Loth KA, MacLehose RF, Fulkerson JA, Crow S, Neumark-Sztainer D. Eat this, not that! Parental demographic correlates of food-related parenting practices. Appetite. 2013;60(1):140-7.

36. Kaplan JB, Bennett $\mathrm{T}$. Use of race and ethnicity in biomedical publication. Jama. 2003;289(20):2709-16.

37. Bower A. African American Foodways; explorations of history and culture. Urbana and Chicago: University of Illinois Press; 2007.

38. Tipton-Martin T. The Jemima Code : two centuries of African American Cookbooks: University of Texas Press; 2015.

39. D.C. Health Matters 2019 [Available from: http://www.dchealthmatters.org/ demographicdata?id=131495\&sectionld=940.

40. Tong TYN, Wareham NJ, Khaw K-T, Imamura F, Forouhi NG. Prospective association of the Mediterranean diet with cardiovascular disease incidence and mortality and its population impact in a non-Mediterranean population: the EPIC-Norfolk study. BMC Med. 2016;14(1):135.

41. Dernini S, Berry EM. Mediterranean diet: from a healthy diet to a sustainable dietary pattern. Front Nutr. 2015;2:15.

42. Monlezun DJ, Leong B, Joo E, Birkhead AG, Sarris L, Harlan TS. Novel longitudinal and propensity score matched analysis of hands-on cooking and nutrition education versus traditional clinical education among 627 medical students. Adv Prev Med. 2015;2015.

43. Monlezun DJ, Kasprowicz E, Tosh KW, Nix J, Urday P, Tice D, et al. Medical school-based teaching kitchen improves $\mathrm{HbA1c}$, blood pressure, and cholesterol for patients with type 2 diabetes: Results from a novel randomized controlled trial. Diabetes Res Clin Pract. 2015;109(2):420-6.

44. Wolfson JA, Bostic S, Lahne J, Morgan C, Henley SC, Harvey J, et al. A comprehensive approach to understanding cooking behavior: Implications for research and practice. Br Food J. 2017;119(5):1147-58.

45. Nielsen L, Riddle M, King JW, Aklin WM, Chen W, Clark D, et al. The NIH science of behavior change program: transforming the science through a focus on mechanisms of change. Behav Res Ther. 2018;101:3-11.

46. Michie S, Ashford S, Sniehotta FF, Dombrowski SU, Bishop A, French DP. A refined taxonomy of behaviour change techniques to help people change their physical activity and healthy eating behaviours: the CALO-RE taxonomy. Psychol Health. 2011;26(11):1479-98.

47. Abuhamdeh S, Csikszentmihalyi M. The importance of challenge for the enjoyment of intrinsically motivated, goal-directed activities. Personal Soc Psychol Bull. 2012;38(3):317-30.

48. CDC-NHANES-DBQ. 2009-2010 Data Documentation, codebook, and frequencies- Diet Behavior \& Nutrition (DBQ_F). 2009-2010 [Available from: https://wwwn.cdc.gov/nchs/nhanes/2009-2010/dbq_f.htm.

49. CDC-NHANES-DBQ. National health and nutrition examination survey, 20152016 Data Documentation, Codebook, and Frequencies. Diet Behavior \& Nutrition (DBQ_l) 2015-2016 [Available from: https://wwwn.cdc.gov/Nchs/ Nhanes/2015-2016/DBQ_l.htm.

50. Farmer N, Powell-Wiley T, Middleton K, Mitchell V, Wallen G. Validation of a cooking habit survey in African-Americans using a cognitive interview focus group. Annals of Behavioral Medicine; 2018: oxford univ press inc journals dept, 2001 evans rd, cary, nc 27513 usa.

51. Josman N, Birnboim S. Measuring kitchen performance: what assessment should we choose? Scand J Occup Ther. 2001;8(4):193-202.

52. Stauffer LM, Fisher AG, Duran L. ADL performance of Black Americans and White Americans on the assessment of motor and process skills. Am J Occup Ther. 2000;54(6):607-13.

53. Michaud P. Development and evaluation of instruments to measure the effectiveness of a culinary and nutrition education program. Washington, DC: Clemson University; 2007

54. Bellg AJ, Borrelli B, Resnick B, Hecht J, Minicucci DS, Ory M, Ogedegbe G, Orwig D, Ernst D, Czajkowski S; Treatment Fidelity Workgroup of the NIH Behavior Change Consortium. Enhancing treatment fidelity in health behavior change studies: best practices and recommendations from the NIH Behavior Change Consortium. Health Psychol. 2004;23(5):443-51.

55. Cohen S, Doyle WJ, Skoner DP, Rabin BS, Gwaltney JM Jr. Social ties and susceptibility to the common cold. JAMA. 1997;277(24):1940-4.

56. Bickart KC, Wright Cl, Dautoff RJ, Dickerson BC, Barrett LF. Amygdala volume and social network size in humans. Nat Neurosci. 2010;14:163.
57. Walker SN, Sechrist KR, Pender NJ. The Health-Promoting Lifestyle Profile: development and psychometric characteristics. Nurs Res. 1987; 36(2):76-81.

58. Cohen S. Perceived stress in a probability sample of the United States; 1988.

59. Diez Roux AV, Mujahid MS, Morenoff JD, Raghunathan T. Assessing the measurement properties of neighborhood scales: from psychometrics to ecometrics. Am J Epidemiol. 2007;165(8):858-67.

60. Ma X, Barnes TL, Freedman DA, Bell BA, Colabianchi N, Liese AD. Test-retest reliability of a questionnaire measuring perceptions of neighborhood food environment. Health Place. 2013;21:65-9.

61. Dubowitz T, Ghosh-Dastidar M, Cohen DA, Beckman R, Steiner ED, Hunter $\mathrm{GP}$, et al. Diet and perceptions change with supermarket introduction in a food desert, but not because of supermarket use. Health Aff (Millwood). 2015;34(11):1858-68.

62. Lorig K, Stewart A, Ritter P, Lynch J, Gonzalez V, Laurent D. Outcome measures for health education and other health care interventions. Washington, DC: Sage; 1996.

63. Buysse DJ, Reynolds CF, Monk TH, Berman SR, DJJPr K. The Pittsburgh Sleep Quality Index: a new instrument for psychiatric practice and research. 1989; 28(2):193-213.

64. Booth M. Assessment of Physical Activity: An International Perspective. Res Q Exerc Sport. 2000;71(sup2):114-20.

65. NCl-Epidemiology and genomics research program. Overview \& Background of The Healthy Eating Index [Available from: https://epi.grants.cancer.gov/ hei/.

66. Papadaki A, Johnson L, Toumpakari Z, England C, Rai M, Toms S, et al. Validation of the English Version of the 14-Item Mediterranean Diet Adherence Screener of the PREDIMED Study, in People at High Cardiovascular Risk in the UK. Nutrients. 2018;10(2):138.

67. McGuire S, Todd JE, Mancino L, Lin B-H. The impact of food away from home on adult diet quality. ERR-90, U.S. Department of Agriculture, Econ. Res. Serv., February 2010. Adv Nutr. 2011;2(5):442-3.

68. Bernstein $M$, Munoz N. Position of the academy of nutrition and dietetics: food and nutrition for older adults: promoting health and wellness. J Acad Nutr Diet. 2012;112(8):1255-77.

69. Ames NJ, Peng C, Powers JH, Leidy NK, Miller-Davis C, Rosenberg A, et al. Beyond intuition: patient fever symptom experience. J Pain Symptom Manag. 2013;46(6):807-16.

70. Billingham SAM, Whitehead AL, Julious SA. An audit of sample sizes for pilot and feasibility trials being undertaken in the United Kingdom registered in the United Kingdom Clinical Research Network database. BMC Med Res Methodol. 2013;13(1):104.

71. Wolfson JA, Smith KC, Frattaroli S, Bleich SN. Public perceptions of cooking and the implications for cooking behaviour in the USA. Public Health Nutr. 2016;19(9):1606-15.

72. Harris PA, Taylor R, Thielke R, Payne J, Gonzalez N, Conde JG. Research electronic data capture (REDCap) - a metadata-driven methodology and workflow process for providing translational research informatics support. J Biomed Inform. 2009;42(2):377-81.

73. Guidance for Clinical Trial Sponsors: Establishment and Operation of Clinical Trial Data Monitoring Committees, US DHHS, FDA. 2006.

74. NIH Office of Behavioral and Social Sciences. Best practices for mixed methods research in the health sciences (2nd ed). 2018.62.

75. Jeffries N, Zaslavsky AM, Diez Roux AV, Creswell JW, Palmer RC, Gregorich $\mathrm{SE}$, et al. Methodological approaches to understanding causes of health disparities. Am J Public Health. 2019;109(S1):S28-33.

\section{Publisher's Note}

Springer Nature remains neutral with regard to jurisdictional claims in published maps and institutional affiliations. 